\title{
EGFR tyrosine kinase inhibitors as first-line therapy in advanced EGFR mutation-positive non-small cell lung cancer: strategies to improve clinical outcome
}

\author{
Andreas Tiefenbacher, Robert Pirker \\ Department of Medicine I, Medical University of Vienna, Vienna, Austria \\ Correspondence to: Prof. Robert Pirker. Department of Medicine I, Medical University of Vienna, Währinger Gürtel 18-20, 1090 Vienna, Austria. \\ Email: robert.pirker@meduniwien.ac.at. \\ Provenance: This is an invited Editorial commissioned by Editorial Board Member Dr. Wenhua Liang (Associate Professor of Thoracic Oncology, the \\ First Affiliated Hospital of Guangzhou Medical University, Guangzhou, China). \\ Comment on: Rosell R, Dafni U, Felip E, et al. Erlotinib and bevacizumab in patients with advanced non-small-cell lung cancer and activating EGFR \\ mutations (BELIEF): an international, multicentre, single-arm, phase 2 trial. Lancet Respir Med 2017;5:435-44.
}

Submitted Sep 19, 2017. Accepted for publication Sep 25, 2017.

doi: $10.21037 /$ jtd.2017.10.02

View this article at: http://dx.doi.org/10.21037/jtd.2017.10.02

The epidermal growth factor receptor (EGFR) has been established as a clinically relevant target for the treatment of patients with advanced non-small cell lung cancer (NSCLC) (1). EGFR blockade can be achieved by either monoclonal antibodies directed against the surface of the receptor or tyrosine kinase inhibitors directed against the intracellular domain of the receptor $(1,2)$. Monoclonal antibodies such as cetuximab and necitumumab improved outcome including overall survival, particularly in patients with squamous cell NSCLC and/or high EGFR expression or EGFR fluorescence in situ positivity (3-7). EGFR tyrosine kinase inhibitors (TKIs) have also been established for the treatment of patients with advanced NSCLC (1). While these TKIs show efficacy in non-oncogene-driven NSCLC, they have much higher efficacy in patients who harbour EGFR mutations in their tumours. EGFR mutations occur in tumours of about $40 \%$ of Asian patients and $15 \%$ of Caucasian patients with advanced NSCLC (8). Based on the results from several randomized phase 3 trials, EGFR TKIs have been established as first-line therapy in patients with advanced EGFR mutation-positive NSCLC (1).

After a median treatment duration of $8-13$ months, patients will develop resistance to EGFR TKIs and clinically progress. In approximately $50-60 \%$ of the resistant patients, resistance is due to the emergence of the T790M mutation (9). In order to improve outcome in these patients, thirdgeneration EGFR TKIs have been developed (10). They include osimertinib, rociletinib and olmutinib. These drugs target both EGFR mutations and the T790M resistance mutation, while they spare wild-type EGFR. These properties should result in enhanced clinical efficacy at lower toxicity in comparison to first- and secondgeneration TKIs. Osimertinib was evaluated in phase 3 trials but the clinical development of the other two agents was halted because of insufficient efficacy or enhanced toxicity. The AURA3 phase 3 trial demonstrated the benefit of osimertinib over chemotherapy in patients with T790M-mediated resistance (11). Osimertinib improved progression-free survival compared to chemotherapy with platinum plus pemetrexed in patients who have acquired T790M-mediated resistance during treatment with EGFR TKIs. Data on overall survival were immature at the time of analysis. Based on these results, osimertinib has become the standard treatment of patients with advanced EGFR mutation-positive NSCLC who have developed T790Mmediated resistance during treatment with EGFR TKIs.

Several strategies have been investigated to improve outcome of first-line treatment with EGFR TKIs in patients with advanced EGFR mutation-positive NSCLC. These include EGFR TKIs combined with either bevacizumab or immunotherapy, and osimertinib as a third-generation EGFR TKI.

EGFR TKIs in combination with bevacizumab have been studied as first-line therapy in patients with EGFR 
mutation-positive NSCLC. The rationale for combining both drugs is two-fold. Firstly, bevacizumab was shown to improve outcome when added to first-line chemotherapy in patients with advanced non-squamous cell NSCLC. Bevacizumab combined with carboplatin plus paclitaxel increased progression-free survival and overall survival, and bevacizumab added to cisplatin plus gemcitabine improved progression-free survival but did not increase overall survival compared to chemotherapy alone $(12,13)$. Secondly, treatment with EGFR TKIs may lead to up-regulation of the expression of vascular endothelial growth factor $(14,15)$. Both reasons led to clinical studies of EGFR TKIs plus bevacizumab in patients with advanced EGFR mutationpositive NSCLC.

The BELIEF trial was a single-arm, phase 2 trial done in 29 European countries (16). The trial evaluated oral erlotinib $150 \mathrm{mg}$ per day plus intravenous bevacizumab $15 \mathrm{mg} / \mathrm{kg}$ every 21 days in 109 patients with stage IIIB or stage IV mutation-positive adenocarcinomas of the lung. The trial also tested whether outcome differs between patients with co-existing T790M mutations and those without T790M mutations. Therefore, patients were stratified according to the presence of the T790M mutation in the tumours. The primary endpoint of the trial was progression-free survival. T790M mutations were detected in $34 \%$ of the patients. Median progression-free survival was 13.2 months for the total population, 16 months for patients with co-existing T790M mutation and 10.5 months for those without T790M mutation. Median survival was 28 months but survival data were still immature at the time of analysis. Toxicity was considerable with $29 \%$ of patients experiencing a serious adverse event. Five grade 4 events (acute coronary syndrome, biliary tract infection, colon perforation, and other neoplasm) and one treatment-related death due to sepsis were observed. The authors concluded that the combined treatment benefits patients.

Japanese investigators have also evaluated erlotinib plus bevacizumab in patients with advanced EGFR mutationpositive NSCLC. In contrast to the BELIEF study, the Japanese study was a randomized phase 2 trial (17). A total of 154 patients were enrolled and randomized in a 1:1 ratio to oral erlotinib $150 \mathrm{mg}$ per day plus intravenous bevacizumab $15 \mathrm{mg} / \mathrm{kg}$ every three weeks or to erlotinib alone. Treatment was continued until disease progression or unacceptable toxicity. Progression-free survival as the primary endpoint was prolonged with the combined treatment. The hazard ratio was 0.54 (95\% CI, 0.36-0.79; $\mathrm{P}=0.0015)$ and median progression-free survival times were
16 and 9.7 months, respectively. Overall survival data were immature at the time of analysis. Serious adverse events occurred at similar rates among both groups (24\% and $25 \%$, respectively). However, grade 3 or worse adverse events were more frequently seen with erlotinib plus bevacizumab than with erlotinib (91\% and 53\%, respectively). Hypertension, haemorrhagic events and proteinuria were more frequent in the bevacizumab group.

The BELIEF study and the Japanese study indicated good efficacy of erlotinib plus bevacizumab in terms of progression-free survival but at the expense of increased toxicity. Based on these results, erlotinib plus bevacizumab has been approved by the European Medicines Agency (EMA) for the first-line treatment of patients with advanced EGFR mutation-positive NSCLC. However, a randomized phase 3 trial is required in order to definitively prove the clinical benefit of this combined treatment in these patients, particularly also in terms of overall survival. Until such a trial confirms the survival benefit, we believe that erlotinib plus bevacizumab can be considered as a treatment option for selected patients with advanced EGFR mutationpositive NSCLC but not as standard treatment.

EGFR TKIs in combination with immune checkpoint inhibitors have been studied as another strategy to improve outcome of first-line treatment with EGFR TKIs. Based on initial clinical trials, however, these combinations resulted in unexpected high toxicity, in particular interstitial lung disease (18). Therefore, further clinical evaluation of these combinations has been halted. In this context it should be noted that immune checkpoint inhibitors appear to have less efficacy in patients with oncogene-driven cancers than in those with non-oncogene-driven cancers. This difference in efficacy may be explained by a much higher tumour mutational burden in smoking-related cancers than in EGFR mutation-positive driven cancers. Thus, the clinical value of immune checkpoint inhibitors in patients with advanced EGFR mutation-positive NSCLC remains yet to be proven.

The most promising strategy to improve outcome of first-line treatment of patients with advanced EGFR mutation-positive NSCLC focussed on third-generation EGFR TKIs. Recently, the FLAURA trial demonstrated the superiority of osimertinib over first-generation EGFR TKIs in the first-line treatment of TKI-naive patients with advanced EGFR mutation-positive NSCLC (19). Osimertinib increased progression-free survival compared to erlotinib or gefitinib. The hazard ratio was 0.30 (95\% CI, 0.23-0.41), and median progression-free survival times 
were 10.1 and 4.4 months, respectively. Response rates were $71 \%$ with osimertinib and 31\% with chemotherapy. Quality of life was also improved with osimertinib, in particular in terms of cough, chest pain, dyspnea, fatigue, and appetite loss. Overall survival data were not reported and are eagerly awaited.

In conclusion, adding bevacizumab to first-line treatment with EGFR TKIs in patients with advanced EGFR mutation-positive NSCLC may improve clinical outcome but, in our opinion, requires further proof of its clinical efficacy in a randomized phase 3 trial before its wide-spread use should be recommended. Combinations of EGFR TKIs with immune checkpoint inhibitors outside of clinical trials are not recommended at this time because of their increased toxicity in early clinical trials. However, osimertinib can be considered as a new standard in the first-line treatment of patients with advanced EGFR mutation-positive NSCLC.

\section{Acknowledgements}

None.

\section{Footnote}

Conflicts of Interest: A Tiefenbacher has no conflicts of interest to declare. $\mathrm{R}$ Pirker has received speaker's fees and honoraria for consulting from AstraZeneca, Boehringer Ingelheim and Eli Lilly.

\section{References}

1. Pirker R. What is the best strategy for targeting EGF receptors in non-small-cell lung cancer? Future Oncol 2015;11:153-67.

2. Pirker R. Epidermal growth factor receptor-directed monoclonal antibodies in nonsmall cell lung cancer: an update. Curr Opin Oncol 2015;27:87-93.

3. Pirker R, Pereira JR, Szczesna A, et al. Cetuximab plus chemotherapy in patients with advanced non-small-cell lung cancer (FLEX): an open-label randomised phase III trial. Lancet 2009;373:1525-31.

4. Pujol JL, Pirker R, Lynch TJ, et al. Meta-analysis of individual patient data from randomized trials of chemotherapy plus cetuximab as first-line treatment for advanced non-small cell lung cancer. Lung Cancer 2014;83:211-8.

5. Pirker R, Pereira JR, von Pawel J, et al. EGFR expression as a predictor of survival for first-line chemotherapy plus cetuximab in patients with advanced non-small-cell lung cancer: analysis of data from the phase 3 FLEX study. Lancet Oncol 2012;13:33-42.

6. Herbst RS, Redman MW, Kim ES, et al. Cetuximab plus carboplatin and paclitaxel versus carboplatin and paclitaxel alone, with or without bevacizumab, in advanced NSCLC (SWOG S0819): a randomised, phase 3 study. Lancet Oncol 2017. [Epub ahead of print].

7. Thatcher N, Hirsch FR, Luft AV, et al. Necitumumab plus gemcitabine and cisplatin versus gemcitabine and cisplatin alone as first-line therapy in patients with stage IV squamous non-small-cell lung cancer (SQUIRE): an open-label, randomised, controlled phase 3 trial. Lancet Oncol 2015;16:763-74.

8. Dearden S, Stevens J, Wu YL, et al. Mutation incidence and coincidence in non small-cell lung cancer: meta analyses by ethnicity and histology (mutMap). Ann Oncol 2013;24:2371-76.

9. Oxnard GR, Arcila ME, Sima CS, et al. Acquired resistance to EGFR tyrosine kinase inhibitors in EGFRmutant lung cancer: distinct natural history of patients with tumors harboring the T790M mutation. Clin Cancer Res 2011;17:1616-22.

10. Pirker R. Third-generation epidermal growth factor receptor tyrosine kinase inhibitors in advanced nonsmall cell lung cancer. Curr Opin Oncol 2016;28:115-21.

11. Mok TS, Wu Y-L, Ahn M-J, et al. Osimertinib or platinum-pemetrexed in EGFR T790M-positive lung cancer. N Engl J Med 2017;376:629-40.

12. Sandler A, Gray R, Perry MC, et al. Paclitaxel-carboplatin alone or with bevacizumab for non-small-cell lung cancer. N Engl J Med 2006;355:2542-50.

13. Reck M, von Pawel J, Zatloukai P, et al. Phase III trial of cisplatin plus gemcitabine with either placebo or bevacizumab as first-line therapy for nonsquamous non-small-cell lung cancer: AVAil. J Clin Oncol 2009;27:1227-34.

14. Fan $W$, Tang Z, Yin L, et al. MET-independent lung cancer cells evading EGFR kinase inhibitors are therapeutically susceptible to $\mathrm{BH} 3$ mimetic agents. Cancer Res 2011;71:4494-505.

15. Wei LH, Kuo ML, Chen CA, et al. Interleukin-6 promotes cervical tumor growth by VEGF-dependant angiogenesis via a STAT3 pathway. Oncogene 2003;22:1517-27.

16. Rosell R, Dafni U, Felip E, et al., Erlotinib and bevacizumab in patients with advanced non-small-cell lung cancer and activating EGFR mutations (BELIEF): an international, multicentre, single-arm, phase 2 trial. Lancet Respir Med 2017;5:435-44. 
17. Seto T, Kato T, Nishio M, et al. Erlotinib alone or with bevacizumab as first-line therapy in patients with advanced non-squamous non-small-cell lung cancer harbouring EGFR mutations (JO25567): an open-label, randomised, multicentre, phase 2 study. Lancet Oncol 2014;15:1236-44.

18. Ahn MJ, Yang J, Yu H, et al. 136O: Osimertinib combined with durvalumab in EGFR-mutant non-small cell lung cancer: Results from the TATTON phase Ib trial. J

Cite this article as: Tiefenbacher A, Pirker R. EGFR tyrosine kinase inhibitors as first-line therapy in advanced EGFR mutation-positive non-small cell lung cancer: strategies to improve clinical outcome. J Thorac Dis 2017;9(11):4208-4211. doi: $10.21037 /$ jtd.2017.10.02
Thorac Oncol 2016;11:S115.

19. Ramalingam $S$, Reungwetwattana $T$, Chewaskulyong B, et al. Osimertinib vs standard of care (SoC) EGFR-TKI as first-line therapy in patients (pts) with EGFRm advanced NSCLC: FLAURA. Presented at: ESMO 2017 Congress; Madrid, Spain; September 9-12, 2017. Ann Oncol 2017;28:v605-49. 